\title{
True Control Status
}

National Cancer Institute

\section{Source}

National Cancer Institute. True Control Status. NCI Thesaurus. Code C99270.

The participant is a true control for the phenotype under consideration. 